\title{
Desarrollo de un marco metodológico del proceso de verificación y validación de software para pequeñas y medianas empresas
}

\section{ABSTRACT}

Hoy en día es complicado para una Pyme desarrolladora de Software aplicar los Modelos de Calidad y Pruebas propuestos, tales como: CMMI, CMM, MOPROSOFT, COMPETISOFT, TMMi, TMap que hacen referencia al proceso de Verificación y Validación (V\&V), principalmente por: 1) el grado de complejidad, la mayoría han sido estructurados para ser aplicados en empresas grandes; 2) las restricciones de costo de las Pymes; 0 3) por el desconocimiento que se tiene sobre los beneficios que se obtendrían. El presente trabajo tiene como principal objetivo elaborar un Marco Metodológico del Proceso de V\&V ajustado a la realidad socio económico de las Pymes, el trabajo incluye una guía metodológica, los roles y competencias del equipo de trabajo, plantillas/listas de verificación base y las principales métricas. El Marco fue aplicado al Sistema de Control de Inventarios de la empresa QBOSS $S A C$, que es una Pyme peruana, obteniéndose mejoras en la calidad del software.

Keywords: calidad, estándares de V\&V, pruebas, V\&V

Desarrollo de un Marco metodológico DEL PROCESO DE VERIFICACIÓN Y VALIDACIÓN DE SOFTWARE PARA PEQUEÑAS Y MEDIANAS EMPRESAS

\section{RESUMEN}

Today it is difficult for an SME to apply the Quality Models and proposed tests such as CMMI, TMMi, TMap, IEEE Standard referring to the process of $V$ \& V, this primarily by: 1) the degree of complexity;; 2) cost constraints that SMEs do not have enough money to hire market specialists that can provide the directives they need or 3 ) simply because of the ignorance that exists about the benefits obtained. The present research has as main objective to develop a methodological framework Process Verification and Validation adjusted for socioeconomic reality of SME. The framework was applied to the project Inventory Control System of the Company QBOSS SAC, the initial situation of the company was analyzed, then the methodological framework developed was applied, and finally the results were positive for the organization.

Palabras clave: standard V\&V, testing, verification and validation, quality

\section{INTRODUCCIÓN}

Los esfuerzos de Verificación y Validación ( $\mathrm{V} \& \mathrm{~V}$ ) tienen como objetivo asegurar que el software satisfaga los requisitos del usuario y la calidad esperada [7]. Para diferenciar ambos términos, Boehm en 1979 en [12] formuló las siguientes preguntas. Verificación: ¿Estamos construyendo el producto correctamente?, Validación: ¿Estamos construyendo el producto correcto?

El proceso de Verificación determina si los productos software de una actividad (o Fase) cumplen con los requisitos o condiciones que tienen impuestas por las actividades precedentes. Estos procesos pueden incluir análisis, revisión y pruebas. Mientras que el proceso de validación determina si los requisitos y el sistema o producto software tal como se ha construido cumplen con su uso específico previsto.

Existe un conjunto de técnicas que se pueden aplicar para la comprobación del software, a la vez, según [10] las clasifica en: Análisis Estático, Análisis Dinámico y Análisis Formal. Análisis estático, las actividades que corresponden a esta clase, no requieren de la ejecución del software para su evaluación. Mientras que en Análisis dinámico, las actividades que corresponden a esta clase, sí se requieren de la ejecución del software para su evaluación. Esta técnica también son conocidas como Pruebas del software. Y Analisis Formal, usa teorías matemáticas para analizar el algoritmo de una solución.

A pesar de contar con modelos, estándares y herramientas enfocada a la Calidad y a los procesos de Verificación y Validación de Software, el resultado no es alentador en las organizaciones, se observan: Productos que no satisface los requerimientos del usuario, planes replanificados $n$ veces, inclusiones de ciclos extraordinarios de pruebas, convocatorias de recursos altamente especializados en etapas finales del proyecto, solicitud de presupuesto adicional, horas extras, recursos y clientes desmotivados o periodos de estabilización que no tienen fin.

Al revisar los modelos y estándares de calidad y pruebas, se observa que, en su mayoría, están estructurados para ser aplicados en organizaciones grandes y medianas, lo que supone una gran inversión en dinero, tiempo y recursos, además los

\footnotetext{
* Magister en Ingeniería de Sistemas. Analista de Sistemas Interbank.

E-mail: lizette.grados@gmail.com

** Doctora en Informática. Docente Asociada UNMSM. E-mail: nlasernap@unmsm.edu.pe
} 
modelos indican qué se debe hacer, pero no cómo implementarlo. En el caso de las Pymes tendrían que contratar expertos para entender qué procesos, métricas, roles, plantillas y checklist son los necesarios para llevar a cabo un adecuado proceso de V \& V del Software. Las restricciones de dinero, tiempo y recursos no permiten implementar un software de calidad en las pequeñas empresas como las Pymes.

Para mitigar esta situación se requiere del compromiso de la alta gerencia y que el proceso de $V$ \& V de software este soportado por un Marco Metodológico flexible acorde con el tipo de organización. El trabajo desarrollado tuvo como objetivo principal elaborar un Marco Metodológico del Proceso de Verificación y Validación ajustado a la realidad socio económico de las Pymes y enfocado en los procesos claves de V \& V. Para ello, se toman como referencia los modelos y estándares de calidad más representativos que existen, se incluyen los roles y competencias del equipo de trabajo, se propusieron las principales métricas para medir la calidad del software, y se elaboraron las plantillas y checklist que deben ser considerados en el Marco.

Para evaluar la bondad del Marco Metodológico, se aplicó al proyecto de Sistema de Control de Inventarios de la empresa QBOSS SAC, que es una Pyme peruana, previo a la aplicación se analizó la situación inicial de la Pyme, luego se aplicó el Marco Metodológico propuesto y finalmente la organización obtuvo resultados positivos con una mejora importante en sus procesos de desarrollo de software, y una reducción en sus tiempos de proceso.

El resto del artículo está organizado de la siguiente manera. En la sección 2 se presenta el Marco Metodológico elaborado, mientras que en la sección 3 se muestra una Aplicación del Marco Metodológico desarrollado. Finalmente, en la sección 4 se presentan las conclusiones y trabajos futuros derivados del presente trabajo.

\section{MARCO METODOLÓGICO DE V\&V PARA PYMES}

El presente Marco Metodológico servirá de guía en los procesos de V\&V del software para las Pymes peruanas. El proceso de $V \& V$ del Software, se inicia con los requerimientos de un proyecto y culmina con la puesta en marcha o pase a producción del software; V\&V no debe ser un proceso aislado que se activa al término de la construcción del Software, sino que debe estar integrado con las necesidades del negocio e ir de la mano en todo el proceso de Desarrollo.

\subsection{Elementos previos a la definición del Marco}

\subsubsection{Modelos y estándares como base}

Se ha tomado en cuenta para elaborar el Marco Metodológico los proceso de $\mathrm{V} \& \mathrm{~V}$ de los modelos y estándares que se indican a continuación, extrayendose lo más adecuado para adaptarlas al Marco. CMMI [1], Competisoft [11], TMMI [14], TMap [13], los estándares IEEE: Std 730-2002 [5], Std 829-1998 [3], Std 1028-2008 [6], Std 1012-2004 [10], Std 610.12-1990 [5], y las Normas ISO/IEC: 9126 [8] [9] [10].

CMMI incluye en el Nivel 3, el área de procesos de $\mathrm{V}$ \& V; Mientras que, Competisoft, define las actividades de $\mathrm{V} \& \mathrm{~V}$ para todos sus procesos. $\mathrm{Y}$ en TMMI, define prácticas específicas de $V$ \& $V$ en las metas de las áreas de proceso. A la vez, en Tmap [8] define las actividades de $V$ \& $V$ en los procesos propuestos. Los estándares ISO/IEC establecen las pautas para la gestión de la calidad de los productos de software, mientras que los estándares IEEE establecen planes y guías de los procesos de $\mathrm{V} \& \mathrm{~V}$ en todo el ciclo de vida del software.

\subsubsection{Roles del Proceso de V \& V}

Se toma como referencia los roles del Tmap en [13], seleccionando los roles básicos y prioritarios que una Pyme necesitaría:

- Líder del equipo de pruebas e inspecciones, es encargado de las tareas de gestión, supervisión y control de los proyectos de $V \& V$ de las aplicaciones asignadas.

- Inspector, es responsable de la elaboración de los checklist y log de inspección, así como la ejecución y supervisión de las revisiones.

- Analista de pruebas, encargado de la elaboración de los diseños funcionales, así como del diseño, ejecución y supervisión de las pruebas funcionales.

- Ejecutor de Pruebas (Testers), Responsable de garantizar la implementación técnica de las tareas de prueba necesarias.

\subsection{3 Áreas que participan en el proceso de V\&V}

A continuación se detallan las áreas y las responsabilidades que deben seguir.

\section{Comité de Negocio}

a) Levantamiento inicial de requerimientos de los usuarios, b) Establecimiento de reuniones para 
el entendimiento de la solicitud del usuario, c) Elaboración de la especificación funcional detallada, d) Planificación y Priorización de las atenciones teniendo en cuenta los objetivos y metas de la organización.

\section{Oficina de Desarrollo}

a) Análisis inicial de la solicitud del usuario,b)

Análisis de Impacto, c) Elaboración de la estimación del esfuerzo y tiempo de atención, d) Elaboración de la Especificación técnica detallada, e) Manual de Configuración e Instalación, f) Construcción de la solución, g) Soporte durante el proceso de pruebas de calidad, h) Elaboración del plan de reversión.

\section{Oficina de Verificación y Validación}

a) Aseguramiento de la alineación y trazabilidad de la solicitud inicial del usuario con los documentos de especificación y construcción de la solución, b) Elaboración de la lista de Verificación Funcional y Propuesta técnica inicial, c) Elaboración de la estimación del esfuerzo y tiempo de atención, d) Establecimiento del Plan de Pruebas de la atención, e) Elaboración de Checklists para la Especificación Funcional y Técnica, f) Diseño de los casos y escenarios de pruebas, g) Consolidado de las evidencias y reporte de defectos, h) Establecimiento de Métricas y Elaboración de informes de avance.

\subsubsection{Fases del ciclo de vida del software y V\&V}

El Marco que se desarrolla considera las actividades de las fases de Planeación, Análisis, Diseño, Pruebas y Ratificación (pruebas que realiza el usuario en un ambiente controlado o ambiente pre-productivo). A la vez se tienen en cuenta los procesos de $\mathrm{V} \& \mathrm{~V}$ estática y dinámica. El proceso de $V$ \& $V$ estática se inicia desde la fase de planeación hasta el diseño, y el proceso de $\mathrm{V} \& \mathrm{~V}$ dinámica abarca las fases de pruebas y ratificación [12].

\subsubsection{Restricciones}

El Marco propuesto no incluye pruebas automatizadas como pruebas de seguridad, usabilidad o performance. Tampoco se incluye la pruebas unitarias o realizadas por el programador.

La guía es elaborada para los procesos generales que mantienen las Pymes; sin embargo, su implementación dependerá de la realidad de cada empresa.

\subsection{Descripción del Marco Metodológico propuesto}

En la figura 1 se presenta el esquema del Marco propuesto, se observa en la primera fila los procesos que realiza el área de desarrollo: Especificación funcional, especificación técnica, estimación de la solución, priorización y planificación, diseño

Figura 1. Esquema del Marco elaborado para V\&V de Software.

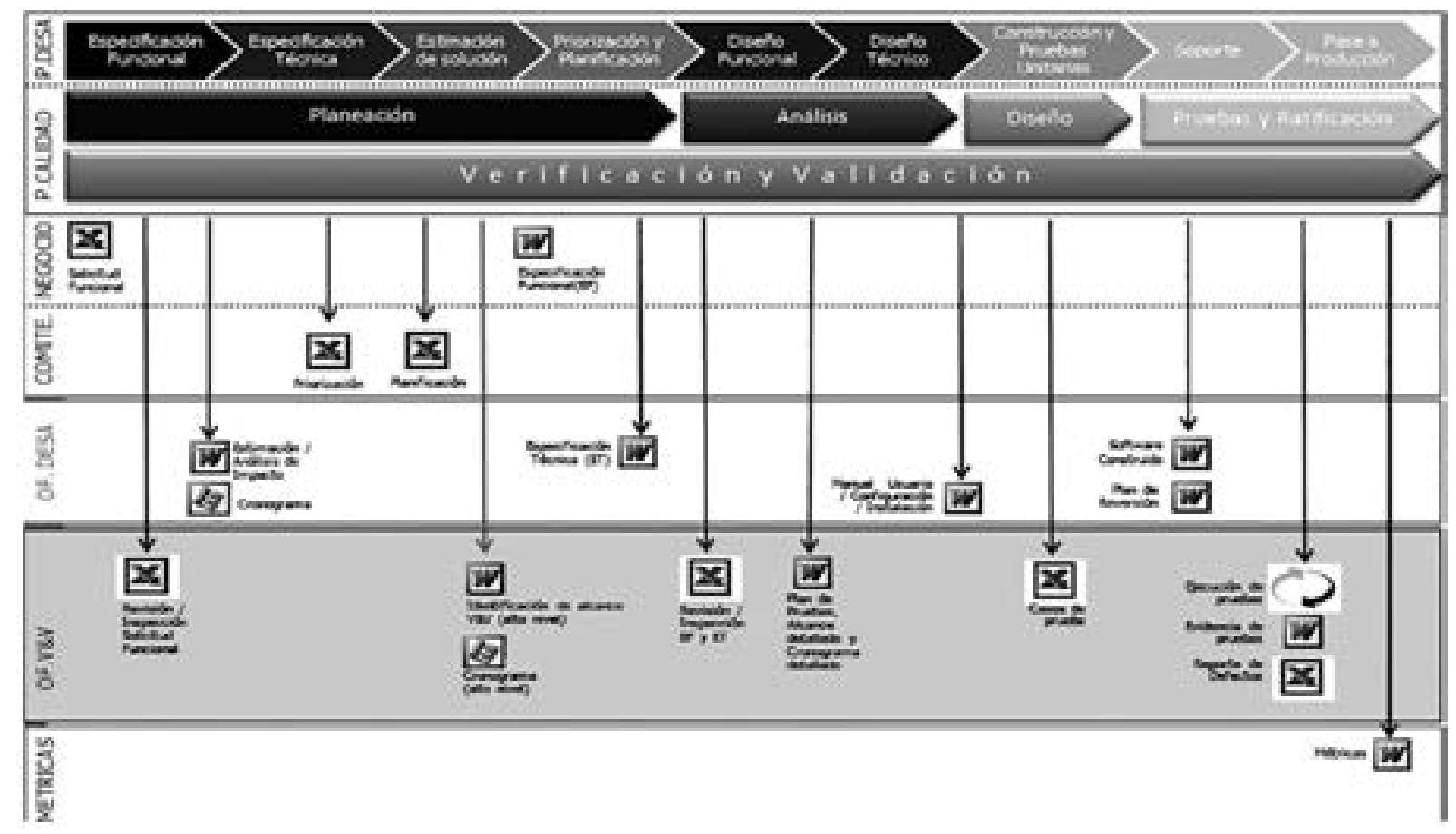


funcional y técnico, construcción y pruebas, Soporte y Post Producción.

Mientras que, en la segunda fila se presentan las fases del ciclo de vida del software a considerar: Planeación, Análisis, Diseño, y Pruebas y Ratificación. A partir de la tercera fila se muestran los entregables de $\mathrm{V} \& \mathrm{~V}$ alineadas a las áreas responsables: El Comité de negocio, el área de desarrollo y el área de V\&V.

A continuación para cada fase del ciclo de vida se describen los procesos de V\&V que se realizan, junto con sus entradas, salidas, y roles que intervienen en los procesos.

\subsubsection{Fase de Planeación}

En esta Fase se identifican dos procesos de V\&V. Primer proceso, se inicia cuando el Comité de Negocio elabora la Solicitud Funcional (documento de entrada), que contiene los requerimientos iniciales del usuario. El área de $\mathrm{V} \& \mathrm{~V}$ realiza el proceso de Inspección y revisión de la solicitud, mediante una lista de verificación o checklist, a la vez se genera un reporte de hallazgos, que el equipo debe hacer el seguimiento para levantar las observaciones con el comité de negocio. El documento de salida de este proceso es la solicitud Funcional verificada.

Segundo proceso, con la solicitud Funcional verificada, el equipo de desarrollo elabora los documentos de Estimación y Análisis de Impacto, y el Cronograma, y el Comité de Negocios evaluará la ejecución del Proyecto, y ejecutará el proceso de priorización y planificación.

En el Equipo de V\&V, el líder de Inspectores y Pruebas elabora los documentos de Identificación de alcance de V\&V y cronograma de alto nivel (documento de salida del proceso).

El equipo de $V \& V$ debe reunir a los equipos de Desarrollo y Comité de Negocios con la finalidad

de asegurar el entendimiento de los requerimientos iniciales, de esta manera la estimación será más certero. Aunque en esta etapa del Proyecto los requerimientos no tienen el suficiente detalle, se debe comunicar al usuario los riesgos y problemas que se pueden ocasionar en la estimación.

\subsubsection{Fase de Análisis}

Esta Fase consta de dos procesos de V\&V.

Primer proceso, a partir de la aprobación de la Solicitud Funcional, se inicia la creación de la Especificación funcional, (junto con los documentos de Identificación de alcance de V\&V y cronograma de alto nivel, forman los documentos de entrada). Contiene los requisitos y la especificación funcional del software. En algunas Pymes el Comité de negocio puede generarlo, y en otras lo elabora el área de desarrollo. El equipo de V\&V, formado por inspectores, revisa mediante un Checklist para la Revisión de Requisitos, y Checklist para la Especificación Funcional. El objetivo es detectar la mayor cantidad de defectos, para que puedan ser levantados por el área que generó el documento. El proceso es cíclico y se repetirá tantas veces como sea necesario. El equipo de desarrollo genera la especificación Técnica. Los documentos de salida son la Especificación Funcional y Técnica Verificada.

Segundo proceso, El equipo de V\&V con el líder del equipo de Inspecciones y Pruebas, a partir de la Especificación Funcional verificada (Entrada) elabora el Plan del Proyecto y el Plan de V \& V (Salida), este último incluye el Plan de Pruebas. El presente Marco Metodológico sólo incluye pruebas funcionales, debido a que este tipo de pruebas es el más solicitado en las empresas y no requiere de una herramienta de automatización.

\subsubsection{Fase de Diseño}

Si el Plan de Verificación y Validación de Software es aprobado, se iniciará el proceso de diseño de pruebas por el analista de pruebas; si no es aprobado tendrá que ajustarse hasta que el Comité de negocio lo apruebe. Los documentos de Especificación Funcional y Técnica verificados serán la entrada para elaborar los escenarios y casos de pruebas (salida), los cuales también deberán ser aprobados por el Comité de negocio. Paralelamente las especificaciones funcionales y técnicas pasarán al área de desarrollo para que se inicie el proceso de Construcción de Software.

\subsubsection{Fase de Pruebas y Ratificación}

Después de que el equipo de Desarrollo termine la construcción del Software, entregará una versión del Software y debe instalarla en el ambiente de pruebas. El equipo de $\mathrm{V} \& \mathrm{~V}$, integrado por el Líder del equipo de Inspecciones y Pruebas y el ejecutor de Pruebas, iniciarán el proceso iterativo de ejecución de pruebas. Los documentos de entrada son el Plan de Pruebas y los Casos de Pruebas, mientras que los documentos de salida son: Reporte de defectos y Evidencias de la ejecución de pruebas. Luego, el equipo de desarrollo deberá levantar los defectos. Una vez que los criterios de calidad se hayan alcanzado el Comité de negocio podrá proceder con la puesta en marcha del software. 
Para cada proceso de V\&V se elabora una tabla, como la que se muestra en la Tabla 1 (Fase de Pruebas, proceso de V\&V: Ejecución de pruebas), en el que se indican los documentos de entrada, salida, roles que intervienen en el proceso, breve descripción del proceso, otros entregables y estándares relacionados.

Tabla 1. Fase de pruebas proceso de V\&V: ejecución de pruebas

\begin{tabular}{|l|l|}
\hline Entrada & $\begin{array}{l}\text { Plan de Pruebas } \\
\text { Casos de Pruebas } \\
\text { Versión Congelada del Software }\end{array}$ \\
\hline Salida & $\begin{array}{l}\text { Reporte de defectos } \\
\text { Evidencias de la ejecución de } \\
\text { pruebas }\end{array}$ \\
\hline Rol & $\begin{array}{l}\text { Líder del equipo de Inspecciones y } \\
\text { Pruebas }\end{array}$ \\
\hline Ejecutor de Pruebas \\
\hline Iniciativa & $\begin{array}{l}\text { El equipo de pruebas iniciará con la } \\
\text { ejecución de los casos de pruebas. } \\
\text { Los entregables de esta etapa son el } \\
\text { reporte de defectos y los documentos } \\
\text { de evidencia. Estos documentos } \\
\text { deberán ser claramente especificados } \\
\text { con la finalidad que el equipo de } \\
\text { desarrollo pueda darle una pronta } \\
\text { solución. }\end{array}$ \\
\hline Estándares \\
Entregables
\end{tabular}

\subsection{Gestión de defectos}

En los Procesos de V\&V estática y dinámica el equipo de Calidad detecta defectos o fallas que deben ser levantados o corregidos por sus respectivos autores, para ello, es importante una adecuada Gestión de Defectos. En [13] se propone una Gestión de defectos que permite planificar, revisar y hacer seguimiento de los mismos de una manera sencilla y ágil. Las actividades deben ser ejecutadas en el siguiente orden: i. Identificar defectos: analizar la lista de inspección para identificar los defectos de la documentación. Este procedimiento se realiza cuando se ejecuta los procesos de V\&V estática. Si se realiza V\&V dinámica entonces se deberá identificar defectos comparando el resultado esperado de los casos de pruebas con el resultado real.

ii. Documentar defectos: documentar el detalle de cada uno de los defectos identificados, incluyendo su prioridad, severidad y la fecha de compromiso para su solución.

iii. Resolver los defectos: llevar a cabo las acciones necesarias para que los autores resuelvan los defectos detectados por el equipo de calidad. Se debe realizar un adecuado seguimiento de los defectos o incidencias hasta el cierre de los mismos, indicando la fecha de cierre y dejando constancia de dicho cierre.

iv. Informar el estado de los defectos: después de resolver los defectos encontrados se procede a informar el estado final de los mismos al responsable del Proyecto.

\subsection{Métricas del Proceso de V \& V de Software}

TMap [13] proporciona listas de métricas útiles para hacer valoraciones sobre la calidad del producto, se propone aquellas que se estimen básicas y mínimas para las Pymes:

- Densidad de Defectos por Módulos. La medición se basa en el principio de pruebas: "Agrupamiento de Defectos". El principio define que un número pequeño de módulos contienen la mayoría de los defectos descubiertos durante el proceso de pruebas. Esta información se sustenta en las diferentes estadísticas y estudios realizados en la industria del software.

- Numero de caminos probados. Proporción entre el número de rutas probadas y el total de rutas existentes. Permite medir porcentualmente la cantidad de rutas que el proceso de V\&V considera probar a través de los casos de pruebas propuestos.

- Efectividad de la detección de defectos. La métrica indica la efectividad del de las actividades de V\&V para hallar defectos. Es el porcentaje obtenido de la cantidad de defectos hallados en las actividades de V\&V y el total de defectos (defectos hallados en V\&V + defectos hallados en producción).

- Presupuesto utilizado en revisiones. Proporción del presupuesto y el coste actual de las pruebas. Será utilizada para estimar el coste 
de implementar las actividades de $\mathrm{V} \& \mathrm{~V}$ en un proyecto.

- Incidencias. Por Tipo de Revisiones y Pruebas, permite medir la efectividad de las actividades de $V \& V$ en cada tipo de revisión.

\subsection{Plantillas utilizadas para las actividades de V \& V}

Se han propuesto plantillas de los documentos de entrada y salida presentadas en las cuatro fases del Marco Metodológico. Dichas plantillas se presentan en [2], por razones de espacio en este artículo solo se presentarán y describirán brevemente.

\subsubsection{Fase de Planeación}

- Solicitud funcional, contiene: Datos generales de la solicitud, esquema de proceso actual y procesos relacionados, descripción de la necesidad, alineamiento estratégico para identificar la prioridad del proyecto dentro de la cartera de negocios.

- Checklist de Inspección, identifica los puntos de la solicitud inicial, se califica el cumplimiento de la verificación de cada ítem, y al final se obtiene el porcentaje de cumplimiento, debe ser $100 \%$.

- Reporte de hallazgos, se describen los hallazgos detectados en el checklist de inspección, contiene columnas como fecha de identificación, severidad, impacto, tipo y estado.

- Plantilla de estimación inicial, para medir factores técnicos y ambientales de la organización.

- Estimación en base a la calificación de requerimientos, según el impacto y flujos asociados.

- Estimación del Proyecto de software, se obtiene a partir de los resultados de los factores técnicos, ambientales y calificar los requerimientos.

\subsubsection{Fase de Análisis}

- Plan de V\&V, el objetivo es establecer las bases para la gestión de $\mathrm{V} \& \mathrm{~V}$.

- Checklist para la Revisión de Requisitos, muestra para cada ítem, si aplica o no aplica.

- Checklist para la Especificación Funcional, muestra para cada ítem, acierto o fallo. Si es fallo describe el error.

- Checklist para la Especificación Técnica, muestra para cada ítem, acierto, no aplica, sin verificar.

\subsubsection{Fase de Diseño}

- Registro de Casos de Pruebas, matriz con los casos de prueba a validar.

\subsubsection{Fase de Pruebas y Ratificación}

- Reporte de ejecución de Pruebas

- Reporte de defectos, lista de defectos encontrados durante la ejecución de pruebas.

\section{APLICACIÓN DEL MARCO METODOLÓGICO}

El Marco Metodológico de V\&V del software elaborado en la sección 2, se aplicó a una Pyme Peruana. A continuación se describen las etapas realizadas: 1 . El análisis de la situación inicial de la empresa; 2. La aplicación del Marco; y 3 . El análisis de los resultados obtenidos.

\subsection{Análisis del estado inicial de la Empresa}

La empresa Q-BOSS, es una PYME peruana, que inicia su operaciones a mediados del 2011 y brinda soluciones informáticas. Actualmente la empresa cuenta con 9 empleados, de los cuales 6 de ellos se dedican a la parte de desarrollo, operación y mantenimiento de los productos de software. La empresa decidió aplicar el Marco Metodológico de V \& V desarrollado.

La gerencia ha decidido apostar por la mejora de procesos para dar soporte a la organización, consolidación y crecimiento de la empresa. A la vez, ofrecer a sus clientes productos y servicios de mayor calidad. La empresa seleccionó el Proyecto de Control de Inventarios para aplicar el Marco Metodológico elaborado. Y la responsable de $\mathrm{V}$ \& $\checkmark$ es la profesional que propone el Marco, que en adelante se mencionará como especialista en V \& V.

En la instalación del proyecto de mejora se recopiló toda la información de la empresa y se firmó una carta de compromiso. Se definió un cronograma general para la aplicación del marco teniendo en cuenta que el tiempo total es de 20 semanas. Previo a la aplicación se realizaron un Análisis del estado inicial de la empresa, y la revisión de sus procesos actuales. Se empleó un promedio de 4 horas por parte del especialista en $V \&$ V y personal de la Empresa en la actividad de instalación del proceso de mejora de la calidad.

Para valorar los procesos se realizó una reunión con la persona responsable de los procesos, debido a que no tenían documentación elaboraron diagramas de actividades de estos procesos. En esta etapa se aplicaron las técnicas de recolección de evidencias 
entrevista y encuestas. El esfuerzo realizado por la empresa fue de 20 horas para esta actividad. En la empresa no se aplica ningún proceso de $\mathrm{V} \& \mathrm{~V}$ estática o dinámica, por tanto las características iniciales de la Empresa son:

- Se consideran cinco fases: planificación, análisis, desarrollo, puesta en producción y soporte.

- Sólo se realizan pruebas unitarias pero ninguna actividad de V\&V de software. Estas pruebas unitarias son ejecutadas por los programadores.

- Se evidenció que en el 89\% de los aplicativos que entran a producción reportan costos de mantenimiento altos.

- Existe un $85 \%$ de llamadas a Help Desk ocasionadas por fallas en el Software.

- Clientes insatisfechos por constantes cortes de servicio.

\subsection{Puesta en marcha del Marco de V\&V en la Empresa}

El primer paso es establecer el alineamiento entre las fases y las áreas involucradas, así como fijar sus responsabilidades y la elaboración de los entregables. La empresa aplicó el Marco elaborado para cada una de las fases. A la vez, se completaron las plantillas y se aplicaron las métricas presentados en la sección 3 . El esfuerzo estimado en este paso por parte de la empresa es de 50 horas.

Es importante mencionar que todas las plantillas completadas para la aplicación del marco se presentan en [2], por razones de espacio no se presentan en este artículo. La fase de Planeación se inició con la solicitud formal del Comité de negocio, para el caso de estudio se realizó una verificación de la Solicitud de Implementación del Software de Control de Inventarios. Esta necesidad fue verificada por el equipo de $V \& V$ para asegurar que el documento se encontraba alineado a lo que realmente necesitaba el usuario, y al cumplimiento de estándares requeridos.

Se completó el checklist de Inspección propuesto. El porcentaje de apego es de $79 \%$, lo que indica que se han detectado incidencias, que origina un reporte de hallazgos. Con la Solicitud Formal Verificada los equipos de desarrollo y $\mathrm{V} \& \mathrm{~V}$ realizaron las primeras estimaciones de esfuerzo y tiempos. Luego, se calificó cada uno de los requerimientos según el número de impactos y números de flujo asociados.

La Tabla 2 muestra la Estimación de la duración del Proyecto Sistema de control de Inventarios en la Fase de Planeación. Se observa que el número de horas estimadas incluyendo los procesos de $V \& V$ es de 469.50 horas, considerando que hay 2 personas que trabajan en el proyecto, que emplean un promedio de 8 horas por día, el número de días estimado es de 29.34 .

La Fase de Planeación termina cuando la solicitud Formal llegó al $100 \%$ de Apego y se obtuvo el alcance y estimación inicial (alto nivel).

La Fase de análisis se inicia con la elaboración del Plan de V\&V para su gestión. El Plan básicamente incluye las revisiones formales de entregables que se elaboraron en esta fase son, el Checklist para la Revisión de Requisitos, el Checklist para la Especificación Funcional (La Tabla 3 muestra parte de la lista) y el Checklist para la Especificación Técnica. Los cuales se encuentran en [2].

En la fase de diseño se elaboró un documento Revisión de Diseño Técnico, el que realiza verificación estática de todos los componentes del Sistema de inventarios: Arquitectura, subsistemas, procesos, integración y otros. Otro documento que se desarrolló en esta etapa es el de Registro de casos de prueba, se elaboraron 15 casos de prueba para los componentes del proyecto.

Tabla 2. Modelo de estimación en la fase de planeación

\begin{tabular}{|c|c|c|c|c|}
\hline \multicolumn{5}{|c|}{ MODELOESTIMACION UCP PARA PROYECTOS DE DESARROLLO } \\
\hline \multicolumn{5}{|c|}{ Sistema de Control de Imventarios } \\
\hline & & & & \\
\hline \multicolumn{2}{|l|}{ Tipo de Estimación } & FEGULAR & & \\
\hline \multicolumn{2}{|l|}{ Grado de Dificultad del Prox } & MEOTO & 10 & \\
\hline \multirow{2}{*}{\multicolumn{5}{|c|}{ FACTORES DE ESTIMACION }} \\
\hline & & & & \\
\hline \multicolumn{2}{|l|}{ Puntos Funcionales sin auste (uUCP) } & & & 48,00 . \\
\hline \multicolumn{2}{|l|}{ Factor de Complejidad Ternica (TCF) } & & & b.76. \\
\hline \multicolumn{2}{|c|}{ Factor de Compleidad de Ambiente [EF] } & & & D.65 \\
\hline \multicolumn{2}{|c|}{ Puntos Funcionales Aiustados (UCP) } & & & 23.56 \\
\hline \multicolumn{2}{|c|}{ Esfuerzo Estimado C1 (Horas/Hombre). } & & & 735,56 \\
\hline \multicolumn{2}{|c|}{ Ciclo 2} & & To\% & 90,69 \\
\hline \multicolumn{2}{|l|}{ Gestión y Stquimiento } & & 60,00 & $\$ 0.00$ \\
\hline \multicolumn{2}{|l|}{ Capactaciones. } & & & 0,0 \\
\hline \multicolumn{2}{|l|}{ Ratificación } & & & 5,0 \\
\hline \multicolumn{2}{|l|}{ Desvisción } & & $60 \%$ & \\
\hline \multicolumn{2}{|l|}{ Esfuerzo Total } & & & 459,50 \\
\hline ANALISIS & $15.00 \%$ & 35,33 & & \\
\hline DISENO & $32,00 \%$ & 70.67 & & \\
\hline CERTIFICACION & $55.00 \%$ & 129.56 & & \\
\hline & $100,00 \%$ & 235.56 & & \\
\hline & & & & \\
\hline Horas Lal $\times$ Recurso & & 8 & & \\
\hline Recursos & & 8 & & \\
\hline Dias Lincales & & 89.34 & & \\
\hline
\end{tabular}


Finalmente, en la Fase de Ejecución y ratificación, después de que el desarrollo del sistema de control de inventarios se concluyó, se inició la ejecución de las pruebas. Esto se realizó después de haber revisado el correcto funcionamiento del ambiente de pruebas y haber obtenido la data con la que se realizaron las pruebas. La ejecución de los casos de pruebas se realizó 3 veces hasta que se lograron levantar todas las incidencias. A la vez, se generó el documento Registro de defectos.

\subsection{Análisis de los resultados de la aplicación}

Se aplicaron las cinco mediciones propuestos en el Marco:

Tabla 3. Revisión de requerimientos funcionales

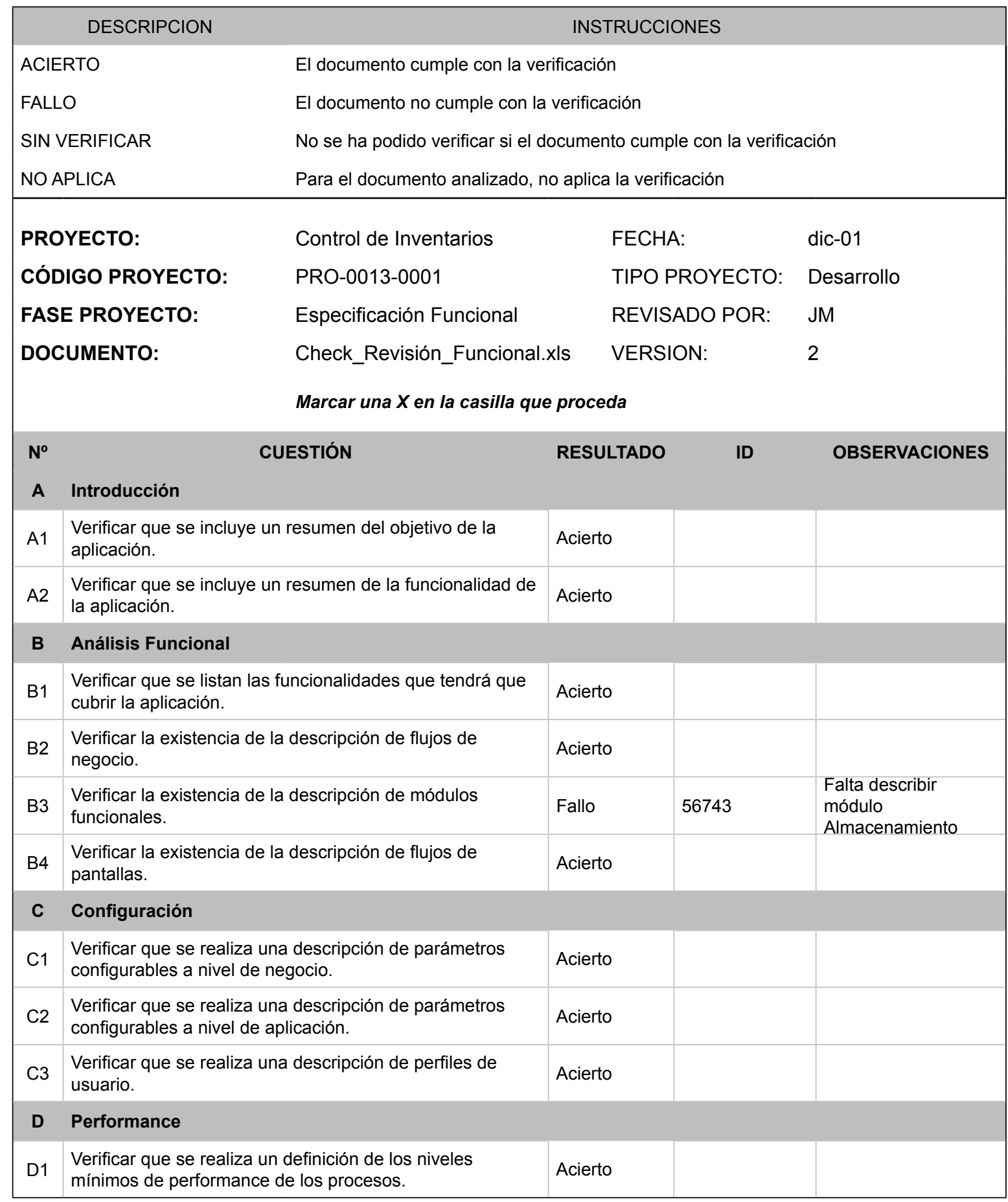


- Densidad de Defectos por Módulos. Esta medición identifica los módulos de la aplicación con mayor cantidad de defectos. Se elaboró un diagrama de Pareto con los datos obtenidos

- Número de caminos probados. Aplicando esta métrica se concluye que hay un $82 \%$ del total de caminos existentes en la aplicación.

- Efectividad de la detección de defectos. No existen incidencias registradas durante el uso del software en el ambiente de producción, se debe al reciente despliegue de la aplicación en producción.

- Presupuesto utilizado en revisiones. El presupuesto invertido en las actividades de revisión alcanzó un $12 \%$ del presupuesto total asignado al proyecto.

- Incidencias Por Tipo de Revisiones y Pruebas

La Figura 2 muestra el porcentaje de incidencias identificados para cada uno de los tipos de pruebas y revisiones hallados en el proyecto de Control de Inventarios. Se observa que el porcentaje de incidencias en los tres últimos tipos de revisiones es bastante bajo especialmente en los de severidad crítica, menos del $2 \%$, mientras que en las pruebas funcionales el porcentaje es alto debido a que cuando se realizan dichas pruebas no hay mucha información del proyecto.

El Proyecto Control de Inventarios se concluyó dentro del tiempo y costos estimados inicialmente. Después de realizar el pase a Producción, se llegaron a detectar incidencias pero no fueron críticos, esto ocurrió porque el ambiente de pruebas en el cual se realizó la certificación del proyecto no tenía las mismas características que el ambiente de Producción.

El costo incurrido por la Pyme en este caso fue cero. El cliente de la empresa se mostró satisfecho con los resultados obtenidos, y los empleados que participaron en el proyecto se mostraron más comprometidos con la implementación de este proyecto, principalmente porque sabían qué procesos ejecutar, el momento en el que deberían hacerlo y las métricas que deberían utilizar. Finalmente debido a la mejora obtenida, la empresa decidió adoptar este Marco Metodológico relacionado a las actividades de Verificación y Validación en la implementación de futuros proyectos de desarrollo.

\section{CONCLUSIONES Y TRABAJOS FUTUROS}

El trabajo ha permitido desarrollar el Marco Metodológico para el proceso de Verificación y Validación de software, ajustado a la realidad socio económico de una Pyme, basado en los modelos y estándares de calidad más representativos del mercado. Asimismo, se incluyó, los roles y competencias del equipo de trabajo, se elaboraron las principales métricas y las plantillas y checklist base que deben ser considerados durante la implementación de un Software de Calidad.

A la vez, se aplicó el Marco Metodológico propuesto durante el desarrollo del Sistema de Control de

Figura 2. Incidencias Por Tipo de Revisiones y Pruebas.

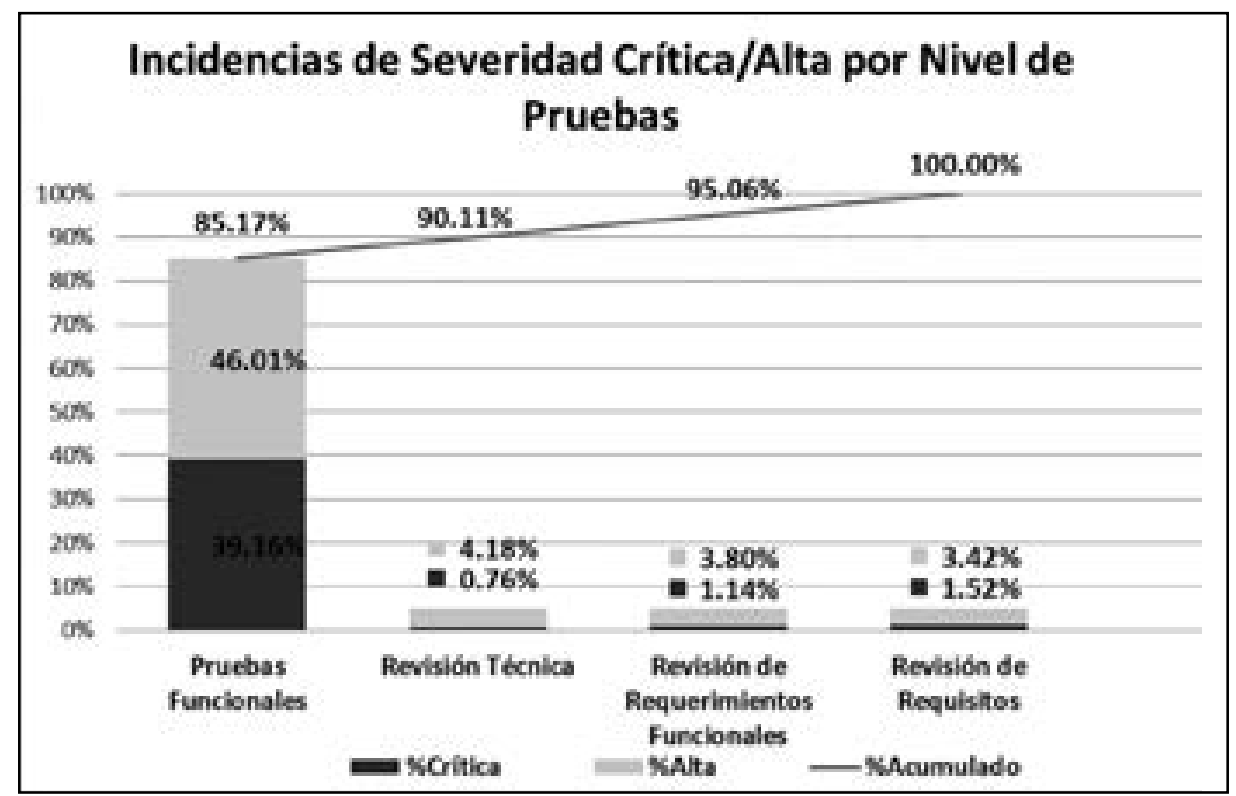


Inventarios de la Pyme peruana QBOSS SAC. Después de aplicar el Marco Metodológico la empresa obtuvo una mejora importante en sus procesos de implementación y mantenimiento de Software. Involucrar al personal y obtener buenos resultados en un periodo de tiempo reducido, fue un aspecto muy importante para aumentar la motivación del personal.

Para la elaboración del trabajo se realizó un amplio estudio de los principales modelos, estándares, normas y trabajos relacionados al Proceso de Verificación y Validación del software.

Trabajos futuros en el tema desarrollado podrían incluir plantillas para diagramas de arquitectura $u$ otros documentos de diseño. $Y$ una guía para la automatización o pruebas de Performance, Seguridad o Usabilidad. Otras investigaciones podrían incluir una guía completa enfocadas en metodologías agiles como XP (Extreme Programing), FDD (Feature-driven developmen) y Scrum.

\section{REFERENCIAS BIBLIOGRÁFICAS}

[1] CMMI for Development (CMMI-DEV) version 1.3. (2010). Software Engineering Institute (SEI), Pittsburg, USA.

[2] GRADOS LIZETTE (2015). Marco metodológico del proceso de verificación y validación de software para pequeñas y medianas empresas. Tesis de Maestría UNMSM.

[3] IEEE Std. 829-1998 Software test documentation. IEEE Computer Society, Software Engineering Technical Committee.

[4] IEEE Std. 610.12-1990, IEEE Standard Glossary of Software Engineering Terminology. IEEE Computer Society, Software Engineering Standard Committee.
[5] IEEE Std 730-2002 Standard for Software Quality Assurance Plans. IEEE Computer Society, Software Engineering Standard Committee.

[6] IEEE Std 1028-2008 Standard for Software Reviews and Audits. IEEE Computer Society, Software Engineering Standard Committee.

[7] IEEE 1012-2004 Standard for Software Verification and Validation. IEEE Computer Society, Software Engineering Standard Committee.

[8] ISO/IEC 9126:2001 Information technology - Software product quality - Part 1: Quality model. Geneva International Organization for Standarization.

[9] ISO/IEC 9126:2002 Software Engineering product quality - Part 2-3: External\&Internal Metrics. Geneva International Organization for Standarization.

[10]ISO/IEC 9126:2004 Software Engineering product quality - Part 4: Quality in use metrics. Geneva International Organization for Standarization.

[11] Oktaba, H.; Piattini, M.; Pino, F.; Orozco, M.J; Alquicira, E. (2008). COMPETISOFT. "Mejora de Procesos de Software para Pequeñas y Medianas Empresas y Proyectos". Madrid España, RA-MA Editorial.

[12]SOMMERVILLE, IAN, Software Engineering 07 Edition, 2005.

[13]TMAP-BASED PROJECT AND PROGRAM TESTING (Consultado 16-5-2015) http://www. sogeti.com/solutions/testing/tmap/

[14]VEENENDAAL, E. (2010). Test Maturity Model integration (TMMi). TMMi Foundation, Ireland. 